\title{
CERTEZA, DUDA ESCÉPTICA Y SABER
}

Samuel M. CabanchiK Universidad de Buenos Aires

¿Se puede decir: Donde no hay duda tampoco hay saber?

Wittgenstein, Sobre la certeza, 121

La valoración del desafío escéptico como una amenaza a nuestras pretensiones de alcanzar algún conocimiento verdadero y fundado acerca del mundo, presenta la naturalidad propia de un lugar común. Y no menos común es encontrar junto a esta valoración un análisis de la noción de certeza que hace de ésta el grado máximo que es dable conquistar en la búsqueda del saber. Luego, según esta tradición, 'certeza' y 'saber' son conceptos solidarios, mientras que la duda escéptica, más allá de las diversas formulaciones e interpretaciones que de ella pudieran hacerse, es el enemigo al que debe combatirse encarnizadamente. Y bien, el presente trabajo se propone desarmar esta trama conceptual, mostrando que 'certeza' y 'saber' no son nociones solidarias sino quizá incompatibles, y propone que la posibilidad de una duda acerca de la supuesta certeza es una condición necesaria para distinguir creencia de saber y, por esta vía, para la construcción de algún saber propiamente dicho.

A fin de lograr este objetivo comenzaremos por analizar la noción de certeza involucrada en algunas posiciones sostenidas por Moore, con ayuda de ciertos instrumentos formales aporta- 
dos por el desarrollo de la lógica epistémica, para luego formular adecuadamente la condición antes mencionada. De esta manera, las discusiones usuales sobre el escepticismo y la certeza recibirán un marco lógico-semántico antes que gnoseológico, aunque el interés de estas páginas es estrictamente filosófico, en el sentido de que en nada contribuirán a la construcción de sistemas formales.

\section{La declaración de certeza}

Desde que Parménides iniciara su viaje por la vía de la verdad, ése ha sido también el camino que muchos filósofos se han propuesto transitar, uno tras otro, con variada suerte. En aquel viejo y venerable poema, no es el filósofo sino una diosa quien traza la dirección de la búsqueda. Supongamos por un momento que Parménides pudiera sospechar de la autenticidad de la diosa o de sus palabras: ino sería "incertidumbre" el nombre apropiado para describir el estado en el que repentinamente se vería sumido? Nuestro filósofo puede ahora pretender relevar por sí mismo a la diosa de su tarea constituyendose en único garante de la verdad, dando lugar así a una declaración de certeza, o bien desafiar a todo caminante empeñado en lograr el acceso a la verdad a que muestre la legitimidad de sus credenciales para ello, asumiendo por su parte que para él ya no hay posibilidad de ninguna garantía: he ahí el desafío escéptico.

La situación imaginaria que se acaba de relatar bien puede tomarse como un símbolo para comprender un proyecto filosofico dominante en la historia de la filosofía, respecto del cual certidumbre e incertidumbre aparecen como dos aspectos complementarios y contrapuestos. Incluso podría decirse que nuestro relato no ha hecho otra cosa que poner a Parménides en la situación de Descartes, por ejemplo. En efecto, también en Descartes es la divinidad quien garantiza la verdad de nuestro conocimiento, y la posibilidad de un engaño celeste no hace 
más que reforzar esta garantía. ${ }^{1}$ Parménides no duda, pero tampoco es él quien tiene a su cargo saber o no saber, sino la diosa; el filósofo sólo transmite sus palabras. Por el contrario, para Descartes es el propio sujeto el que sabe o no sabe. Sus palabras no son las de Dios, por lo que se ve impelido a ofrecer el criterio de verdad que antes era recibido pasivamente. Desde entonces, en cierto sentido, el estado de las cosas no ha variado demasiado. En el presente siglo la cuestión del escepticismo y la certeza fue notablemente reavivada por el filósofo británico George Edward Moore. A continuación seguiremos el desarrollo que ha hecho del asunto este filósofo en diversos artículos.

Una de las tres condiciones que según Moore debe satisfacer su "prueba del mundo exterior", la segunda de ellas para ser más preciso, es conocer con absoluta certeza la verdad de sus premisas. Por lo demás, como explícitamente lo reconoce en el mismo artículo, esta certeza no deriva de una demostración independiente de esas premisas, pues afirma conocer la verdad de las mismas sin poder demostrar o justificar ese conocimiento. "Puedo conocer cosas que no puedo demostrar", nos dice. Y agrega: "Entre las cosas que conozco con certeza, aunque no pueda (según pienso) demostrarlas, estaban las premisas de mis dos pruebas."2 Las premisas aludidas son "aquí hay una mano" más el gesto de ostención y "aquí hay otra mano" más el correspondiente gesto de ostención. No discutiremos ahora la validez o naturaleza de la prueba de Moore, aunque es mucho lo que habría que decir acerca de ello. ${ }^{3}$ No

1 Un comentario en el mismo sentido puede encontrarse en Luis Villoro, op. cit., pp. 142 a 144.

2 G. E. Moore, op. cit., p. 150.

3 Determinar la naturaleza de la "prueba" es, obviamente, una tarea previa a su evaluación. En particular, se trata de aclarar si estamos frente a una demostración de alcance empírico o exclusivamente conceptual. Barry Stroud, op. cit., ha sostenido, contra Ambrose y Malcolm, que es de carácter empírico. Una de las razones que da en favor de su apreciación, es que el propio Moore lo consideraba así. Esto no parece, ciertamente, una buena razón, pues confunde el lugar que un argumento tiene $\rightarrow$ o puede tener- en un texto determinado, con lo que el autor de dicho texto opina sobre él. Pero las opiniones de un autor sobre su producción 
obstante, no podemos dejar de advertir que es al menos extraño incluir en las premisas, que son entidades lingüísticas, los gestos de ostención, que obviamante no lo son. Además, el uso de "otra" en la segunda introduce, por así decir, una referencia de orden temporal que merecería algunas aclaraciones, cosa que Moore no hace en ese texto, aunque sí aparecerá explicitado en el trabajo que ocupará inmediatamente nuestra atención. Supongamos, con todo, que los enunciados o proposiciones $^{4}$ referidos fueran claros. Cabe ahora preguntarse si hay alguna característica que éstos compartan y sea especialmente relevante para la cuestión de la declaración de certeza que se ha hecho acerca de ellos. Esto nos lleva a ese otro artículo de Moore al que antes aludíamos. ${ }^{5}$

no tienen, en principio, ningún privilegio respecto de la de los demás, quizá todo lo contrario. No nos importa lo que Moore quiso decir ni lo que cree que dijo, sino lo que, según cierto punto de vista, efectivamente dijo. Y, precisamente, para mí, no resul ta claro qué quiere decir que la prueba en cuestión es empírica. Por otra parte, aunque presentan importantes diferencias entre sí, Ambrose y Malcolm toman la prueba de Moore de modo completamente distinto a Stroud. Coinciden justamente en que, evaluado desde el punto de vista de sus pretensiones explícitas, el argumento de Moore no refuta al escéptico, en razón de que la posición escéptica plantea una cuestión lógica, no empírica. En consecuencia, la "prueba” de Moore debería tomarse como parte de una estrategia argumentativa dirigida a formular ciertas observaciones conceptuales sobre usos y significados lingüísticos. Aunque esta perspectiva me parece más adecuada que la de Stroud, creo que hay que mostrar cómo la "prueba" de Moore es una cristalización o condensación de un juego filosófico más amplio, pródigo en observaciones gramaticales en el sentido de Wittgenstein. Aun cuando en el presente trabajo no se tematiza específicamente la prueba del mundo exterior ofrecida por Moore, las discusiones desarrolladas en torno a la certeza y al saber constituyen el núcleo de ese juego filosófico que, en última instancia, provoca la aparición de la "prueba". Para una profundización de esta cuestión, puede consultarse el capítulo III del trabajo de Stroud y los artículos de Malcolm y Ambrose citados en las referencias bibliográficas.

4 El hecho de hablar indistintamente de enunciados o proposiciones es, en el presente contexto, más bien una cuestión de estilo que filosófica. En efecto, nuestra discusión no involucra la cuestión ontológica acerca del tipo de entidad que es objeto de la creencia. Este asunto se vuelve importante cuando las expresiones usadas para aludir a esas entidades están ligadas por un cuantificador, pero no tiene mayor relevancia cuando el problema focalizado es el de la lógica de las nociones mismas de "certeza", "saber" y "creencia", fundamentalmente tal como han sido tratadas por los filósofos. Es, en parte, la diferencia entre usar las modalidades como de dicto o de re.

5 Este artículo, "Certainty", fue especialmente escrito por Moore para la an- 
En "Certainty" ["Certeza"], Moore presenta un número de enunciados a los que caracteriza como contingentes, esto es, según sus palabras, una "abreviatura de la expresión larga "proposición que no es autocontradictoria y cuya contradictoria tampoco lo es" ". ${ }^{6}$ De acuerdo con un uso muy extendido en la bibliografía pertinente, nos serviremos de la letra esquemática ' $p$ ' para referirnos al tipo de enunciados de los que contiene casos posibles la lista de Moore. Otra manera de explicitar el significado de la expresión “ ' $p$ ' es contingente”, tanto según lo que dice Moore como según la lógica modal, es analizarla en esta otra: “ ' $p$ ' es posible y 'no $p$ ' es posible". Digamos finalmente que en lo que sigue utilizaremos siempre el estilo indirecto para expresar las formas de enunciados que nos ocupan. ${ }^{7} \mathrm{La}$ aseveración básica y central de Moore por la que deberá comenzar el análisis, es:

\section{(1) "Sé con certeza que p"}

Ahora bien, Moore intenta aclarar y justificar su declaración de certeza a partir de ciertas consecuencias que extrae del hecho de que ' $p$ ' es una proposición contingente. Pero mientras que 'contingente' es respecto de ' $p$ ' una modalidad alética, 'saber' es un operador epistémico. Luego, el tratamiento de la cuestión se desarrollará en el cruce de ambos tipos de modalidades. Consideremos una por una las consecuencias que extrae Moore

tología de la que forma parte (Moore, 1959), y según nos informa el propio Moore, no se trata sino de una conferencia que había dictado ya en 1941. Allí mismo, en el prefacio, Moore aclara que hay en el artículo errores graves que, sin embargo, no sabe cómo corregir. No es seguro que, como opina Casimir Lewy en nota a pie de página, se refiera exclusivamente a los cuatro últimos párrafos.

${ }^{6}$ Moore, op. cit., p. 230.

7 Téngase en cuenta la siguiente observación de Von Wright: “es importante distinguir entre dos interpretaciones de la frase 'se sabe (conoce, verifica) que $a$ ':

“i) 'de la proposición expresada por a se sabe (se conoce) que es verdadera (verificada)', y

“ii) 'se sabe (se conoce, se verifica) que $a$ expresa una proposición verdadera ( $a$ es una variable oracional)' '. Von Wright, op. cit., p. 55.

El sentido en que la usamos aquí es el primero de los citados. 
en relación con la contingencia de los enunciados que captan su atención.

En primer lugar, nos dice que "aunque fuera un hecho que no se conoce nunca la verdad de una proposición contingente, con todo, esto no se seguiría nunca del mero hecho de que es contingente".8 Esta observación es trivialmente verdadera, si se tiene en cuenta la distinción de modalidades. En efecto, como lo señala Von Wright, 'contingente' es a las modalidades aléticas lo que 'no-decidido' es a las epistémicas, 9 por lo que la indeterminación del valor de verdad de ' $p$ ' no permite decidir nada acerca de la posibilidad o imposibilidad de saber que $p$. Lo que ocurre es que hay un uso alético y un uso epistémico de 'posible'. Moore estaría distinguiendo implícitamente entre ambos usos, aunque, como en seguida veremos, luego los confunda. Tiene razón en lo que afirma, pues de "es posible que $p$ y es posible que no $p$ ", no se sigue "no puedo saber que $p$ " y tampoco "no sé que $p$ " sino "no sé si $p$ " que, siguiendo, a Hintikka, interpretaremos como "no sé que $p$ y no sé que no $p$ ". 10

En segundo lugar, Moore señala que, "del hecho de que sean contingentes no se sigue la posibilidad de que sean falsas". " $\mathrm{Si}$ se entiende aquí 'posibilidad' en sentido alético, es claro que se trata de una franca contradicción con la afirmación de que $p$ es contingente. Pero, por lo que unas líneas más abajo sigue diciendo Moore, parece usar ahora 'posibilidad epistémica'. En efecto, pues afirma que "uno al menos de los modos en que usamos expresiones del tipo 'es posible que $p$ ' indica que el enunciado en cuestión no puede ser verdad si la persona que lo formula sabe con certeza que $p$ es falso". ${ }^{12}$ Y esto es, para cierto análisis de 'saber', perfectamente correcto ya que, en ese

\footnotetext{
8 Moore, op. cit., p. 231.

9 Von Wright, op. cit., p. 59.

10 Hintikka, op. cit., p. 12.

11 Moore, op. cit., pp. 231 y 232.

12 Idem, p. 232.
} 
análisis, de "sé que $p$ " se sigue que $p$ es el caso y, en consecuencia, desde el punto de vista de mi saber, no es posible que $p$ no sea el caso. ¿Pero excluye este hecho la posibilided de no $p$ desde el punto de vista alético? La diferencia modal establecida impide una respuesta afirmativa, $y$ todo parece indicar que la intención de Moore lo lleva a confundir ambos sentidos de 'posibilidad'.

La tercera consecuencia nos acerca aún más al nudo del problema. Establecidas las dos anteriores, Moore asume ahora que el hecho de que $\boldsymbol{p}$ sea contingente no es sólo compatible con mi saber acerca de su verdad, sino con mi absoluta certeza de ello. ${ }^{13}$ Aunque no muy claramente, a continuación intenta distinguir entre "sé que $p$ " y "sé con absoluta certeza que $p$ ", y lo hace formulando la siguiente proposición: " "sé que $p$ " implica 'sé con absoluta certeza que $p$ ". ${ }^{14}$ (La palabra "absoluta" parece tener un sentido meramente enfático, por lo que el consecuente no es otro que nuestro enunciado (1).) Esta afirmación es al menos curiosa, y por el momento no tenemos los elementos necesarios para emprender su análisis, pues supone considerar previamente el significado de (1). Antes de entrar de lleno en esa tarea, terminemos con la serie de consecuencias. En rigor, ś́lo queda una, y no es exactamente una consecuencia de la naturaleza contingente de ' $p$ ', sino una observación directamente relacionada con (1), a saber: "del hecho de que sienta la certeza de, o sepa con certeza algo dado, nunca se sigue que cualquier otra persona sienta la certeza de, o sepa la cosa en cuestión; ni del hecho de que él sí, se sigue que yo también". ${ }^{15}$ Estas dos áltimas observaciones sólo se volverán comprensibles una vez interpretado (1); así, vayamos a ello.

En función de una mayor fluidez y simplicidad expositivas, y quizás también de precisión, utilizaremos algunos elemen-

\footnotetext{
13 Idem, pp. 235 y 236.

14 Idem, p. 236.

15 Idem, p. 240.
} 
tos formales de la lógica epistémica, adaptando la notación de Hintikka a nuestro idioma. (Así, por ejemplo, el operador ' $K$ ' de Hintikka será nuestro operador ' $S$ '. Toda vez que sea necesario introducir algún nuevo operador, se hará la aclaración del caso.) ${ }^{16}$ Ahora escribiremos "yo sé que $p$ " de la siguiente manera: " $S_{Y} P$ ", donde el subíndice ' $Y$ ' de ' $S$ ' simboliza el pronombre personal 'yo'. Ahora bien, el operador ' $S$ ' modaliza a ' $p$ '. Puesto que, por todo lo visto, no puede suponerse que Moore use 'certeza' como un recurso retórico, cabe preguntarse: ¿modaliza 'certeza' directamente a ' $p$ '?, ¿̇lo hace pero indirectamente?, ¿o afecta exclusivamente a ' $S$ '? En los sistemas de lógica epistémica no hay un operador especial para traducir la certeza, aunque el operador de creencia suele interpretarse en el sentido de convicción en grado máximo. ${ }^{17}$ Veamos qué nos dice Hintikka al respecto.

Según Hintikka, (1) puede ser un "significado residual" del siguiente enunciado: ${ }^{18}$

(2) " $S_{Y} S_{Y} P$ "

Pero para este autor, (2) es virtualmente equivalente ${ }^{19}$ a " $S_{Y} p$ ", esto es, se implican mutuamente. Aun cuando pudiera resultar discutible esta equivalencia, no puede ser éste el significado de la proposición de Moore. En efecto, como también afirma Hintikka, " $S_{a} S_{b} p \rightarrow S_{a} p$ " es autosuficiente. ${ }^{20}$ Pero entonces, si $a$ soy yo, y lo que sé es que $b$ sabe que sabe $p$, también en este caso sabré $p$. Esto muestra simplemente que el saber es algo

${ }^{16}$ Hintikka, op. cit., p. 11.

${ }^{17}$ Galván, op. ciu., por ejemplo.

${ }^{18}$ En el lenguaje simbólico de Hintikka seńa " $K_{a} K_{a} p$ ”. Para el tema de los significados residuales de esta fórmula, véase op. cit., pp. 116-123.

19 Idem, p. 104. Según Hintikka, ' $p$ ' implica virtualmente ' $q$ ' cuando ' $p \rightarrow q$ ' es autosuficiente, que significa, en el contexto de la lógica epistémica de este autor, que es una oración válida. En consecuencia, el bicondicional " $S_{Y} S_{Y} p \equiv S_{Y} p$ " es válido.

20 Ver nota anterior. 
transmisible. ${ }^{21}$ Mas, ¿no habíamos visto antes que Moore negaba esta transmisibilidad a la certeza? Por lo demás, la iteración de 'saber' no alcanza para dar cuenta de la peculiar fuerza con la que Moore usa la palabra 'certeza'. Más bien parece que 'certeza' está en (1) para modalizar la forma en que el operador ' $S$ ' afecta a ' $p$ '. En este sentido, la interpretación de (1) sería la siguiente:

$$
\text { (3) “ } N S_{Y} p \text { ", }
$$

que se lee: "yo sé necesariamente que $p$ " o "mi saber acerca de que $p$ es necesario" ( $N=$ necesario). En esta interpretación, ' $p$ ' no pierde su carácter contingente, pero en cambio ' $S$ ' adquiere una necesidad que no parece aún justificada; y de hecho Moore no ofrece aclaración alguna de la fuerza que 'certeza' tiene en su uso y nosotros intentamos comprender. En realidad, (3) es la relación entre el operador alético ' $N$ ' y el enunciado " $S_{Y} p$ ", y esto significa que (1) estaría afirmando que no es posible que no sepa que $p$. Es imprescindible ahondar en esta cuestión.

Pensar que la verdadera forma de (1) es la del enunciado (2), tiene la ventaja de ser compatible con la afirmación de Moore de que " $S_{Y} p$ " implica " $S_{Y}$ con certeza $p$ ". Además, Moore no hubiera tenido inconveniente en aceptar el orden inverso del condicional. Bastaría ahora asumir la intransmisibilidad de la certeza como un error de Moore que no invalida los otros aspectos de su posición. Sin embargo, ¿qué se hace en este caso de la certeza? Pues (2) no agregaría nada sustancial al enunciado " $S_{Y} p$ ". Sería una interpretación tan débil de (1) que produciría la desaparición misma del núcleo de la declaración de certeza. Por otra parte, con la interpretación dada por (3) no se resuelve el problema de la transmisibilidad, y se vuelve aún más extraña la implicación de Moore antes formulada. En efecto, ¿cómo aceptar que " $S_{Y} p$ " implica " $N S_{Y} p$ "? Si se la piensa cuanti-

21 Idem, p. 61. 
ficada universalmente, se apreciará cuán inaceptable resulta. Sin embargo, así se conserva la fuerza de 'certeza'. ¿Y si lo que falla aquí es seguir aceptando que Moore en realidad se está refiriendo efectivamente a una forma de saber? Después de todo, afirmar que se trata de una forma de saber y que no obstante no es transmisible, es probablemente inaceptable. ¿Y si se trata de 'creencia' y no de 'saber'? Veámoslo.

Como lo ha observado Hintikka, el hecho de que el principio de transmisibilidad no valga para la lógica de la creencia de la misma forma que para la lógica del saber, se debe básicamente a que, mientras que " $S_{a} p \rightarrow p$ " es verdadero, " $C_{a} p \rightarrow p$ " no lo es. (' $C$ ' $=$ operador ' $B$ ' de Hintikka, que simboliza a 'creencia'. ${ }^{22} \mathrm{Si}$ interpretamos (1) en términos de creencia, sea en la forma de (2) o de (3), comprenderemos por qué no es transmisible como lo es el saber, pero ' $p$ ' vuelve a ser posiblemente falsa, cosa que Moore pretendía evitar. Hay, sin embargo, un enunciado que es demostrado como teorema por Galván ${ }^{23}$ y que expresa, quizá, algo muy cercano a lo que afirma Moore. Es como sigue:

$$
\text { (4) " } C_{Y}\left(C_{Y} p \rightarrow p\right) \text { " }
$$

Es el enunciado que sólo puedo afirmar si soy un sujeto infalible, digamos Dios, pues dice que, dada una creencia mía, creo que la misma es verdadera, esto es, creo que sólo creo verdades. (4) puede ser reforzado complementándolo con

$$
\text { (5) " } C_{Y} p \rightarrow C S_{Y} p \text { " }
$$

que se lee: "si creo que $p$, entonces creo que sé que $p$ ", cuyo significado sería, en última instancia, que no tengo dudas acerca de la veracidad de mis creencias.

Es claro que ni (4) ni (5) dan una garantía objetiva de verdad. Simplemente manifiestan mi imposibilidad de.dudar de la 
verdad de mis creencias, 0 , dicho de otra manera, el hecho de que no puedo dejar de creer que lo que creo es cierto. He aquí el contenido de necesidad que veíamos antes. ¿Pero constituye esto un saber? Cabe recordar ahora lo que Moore decía acerca de las premisas de su prueba del mundo exterior. Según él, esos enunciados los conocía como verdaderos pero sin fundamentos, como el teorema de Gödel, que es verdadero, y a pesar de saber que lo es, no podemos demostrarlo en el sistema. Y bien, creo que (4) y (5) hacen justicia a la declaración de certeza de Moore, $y$ evidencian de esta manera que el intento de garantizar un saber incontrovertible que nos permita un acceso seguro a la verdad, por este procedimiento, no se logra. Los gritos temerarios del dogmático no amedrentan al escéptico, que aún espera que se le entregue la verdad perdida o, al menos, que se le indique qué camino debe tomar para encontrarla.

\section{Certeza y saber}

Dijimos que la declaración de certeza no lograba enunciar las condiciones para garantizar un saber objetivo. Sin embargo, esta afirmación todavía espera su justificación. Para lograrla, es conveniente poner en claro los contrastes esenciales entre la situación de certeza y la de saber. Nuestros enunciados (4) y (5) tratan a la certeza como una forma de creencia y no de saber. Pero al mismo tiempo muestran que, para el sujeto logico ${ }^{24}$ de esa declaración, creencia y saber son lo mismo; "certeza" es, precisamente, el nombre para esta identificación. Dicho de otra manera, nosotros negamos esa identidad, ¿puede negarla el sujeto de la certeza?

Antes de intentar una respuesta directa, debemos volver al artículo de Moore. Allí encontramos la siguiente reflexion: "si digo 'es cierto que $p$ ', el que sepa que $p$ es verdadera es una

24 La expresión "sujeto lógico" pretende indicar, simplemente, que se prescinde aquí de toda caracterización específica de aquel a quien se le atribuye la creencia. El agente queda reducido así a una posición formalmente determinada por la lógica del enunciado mismo. 
condición no sólo necesaria, sino también suficiente de la verdad de mi afirmación. Pero si digo 'no es cierto que $p$ ', entonces el que no sepa que $p$ es verdad, aunque necesaria, no es una condición suficiente de la verdad de mi afirmación". 25 Para captar mejor el sentido de esta observación, téngase en cuenta que para Moore, a pesar de las apariencias, "es cierto que $p$ " involucra tanto al sujeto como "estoy cierto de que $p$ " o "tengo la certeza de que $p$ ". En este pasaje, Moore afirma al menos dos cosas. En primer lugar, establece la equivalencia entre certeza y saber; en segundo lugar, muestra que esta equivalencia se rompe sólo con la introducción de la negación respecto de mi certeza, lo que también puede expresarse diciendo que sólo cuando puedo dudar de mi certeza, ésta y mi saber no son lo mismo. Pero como hemos visto, y para decirlo cartesianamente, mientras creo que $p$, mi certeza y mi saber se confunden todo lo que dure mi creer. En otras palabras, en este sentido de "creer", “ "creo que $p$ ' y 'no sé si $p$ '" es contradictorio en el mismo momento. La declaración de certeza supone así un sujeto compacto - sin fisuras- $e$ intemporal. En efecto, "creo que $p$ " no es incompatible con que en el futuro ' $p$ ' sea falsa para mí, y de igual forma, respecto de una creencia pasada, puedo decir "yo creía que $p$ pero no era cierto que $p$ ". Por otra parte, para que alguna diferencia o fisura sea introducible en el instante presente, debe haber algún otro respecto del cual pueda decirse: "yo creo que $p$ pero él no" o bien "yo estoy cierto de que $p$ pero él (o tú) no sabe(s) si $p$ ". En consecuencia, si yo puedo distinguir en el instante presente entre mi certeza y mi saber, es porque puedo ver mis razones como podría verlas otro. ${ }^{26}$ Entonces, para negar mi certeza es condición necesaria que, o bien haya efectivamente algún otro respecto de mí en tanto sujeto de certeza, o bien yo mismo pueda asumir respecto de mí el punto de vista del otro, sea en relación con el presente

Wittgenstein, op. cit., parágrafo 96.

Villoro, op. cit., p. 141. 
o con otra dimensión temporal. Para enfrentar ahora la cuestión de si estábamos justificados al afirmar que la posición de Moore no lograba asentar un saber objetivo, es necesario mirar más de cerca la noción misma de saber.

Desde que Platón definió 'saber' como "creencia verdadera según razones", ${ }^{27}$ más allá de la riqueza y profundidad que han ganado las discusiones al respecto, la situación no ha variado en lo esencial. En la definición de Platón aparecen tres palabras claves: "creencia", "verdad" y "razones". Ahora bien, mientras que no hay acuerdo sobre si la segunda es o no una condición para saber, y es difícil encontrar una respuesta satisfactoria a la cuestión de cómo y de qué tipo han de ser las razones, hay en cambio cierta coincidencia en incluir "creencia" en la definición de "saber". ${ }^{28}$ Consideremos ahora las relaciones del saber con la creencia. Independientemente de si concebimos a la creencia como un acto de conciencia u ocurrencia mental, o por el contrario, como una disposición a conducirse de determinada manera, supongamos o no a esa disposición correlacionada con un estado interno sujeto a investigación empírica, lo cierto es que la situación de creencia entraña una relación con la verdad, pues en el sentido general en el que ahora tomamos el concepto, 'creencia' significa, al menos parcialmente, 'tener por verdadero'. En esta última frase, hay que distinguir dos elementos: uno, sugerido por el verbo 'tener', que supone una relación con lo que podríamos llamar 'el contenido proposicional' del enunciado ' $p$ '; otro, la creencia en sentido restringido, que consiste en la aseveración del enunciado. Esta distinción se hace necesaria para comprender el significado de la epojé propia de lo que se ha dado en llamar 'escepticismo pirrónico'. En efecto, lo que la epojé interrumpe es la conexión entre el contenido proposicional y la aseveración. Lo que interesa indagar ahora es si para el sujeto

27. Platón, Teetetó, 200-201. (1974).

28 Vilłoro (1986), Hintikka (1962), Galván (1986), Lenzen (1978), Lehrer 
de la creencia tener razones es una condición necesaria de la misma. Y bien, respecto de esta cuestión aparece una diferencia fundamental entre la creencia y el saber, pues cuando digo 'creo que $p$ ', no me comprometo a dar razones, ya que no es cierto que si no tuviera razones para creer que $p$, entonces no podría creer que $p .^{29} \mathrm{Es}$ verdad que si soy un sujeto racional no sostendré creencias de las que no pueda dar cuenta, mas es un hecho que solemos creer sin razones para ello. Pero al margen de la cuestión de hecho, la ausencia de necesidad de razones es de orden conceptual. Por otra parte, sí cabe afirmar que es posible dar razones. Estas razones pueden ser la percepción directa del estado de cosas figurado por el contenido proposicional, las relaciones lógicas del enunciado en cuestión con otros enunciados en los que creo, o simplemente el testimonio de los otros. Que las razones no son una condición necesaria para creer, puede verse en el hecho de que, ante la afirmación 'creo que $p$ ' de algán sujeto, no tendría sentido que se dijera: 'demuéstrame que lo crees dándome tus razones'.

Por el contrario, para saber sí debo tener razones, en el sentido de que si no tengo razones, entonces no sé. Así como, para $m i$, yo no tengo primero razones y después creo, sino que puedo creer independientemente de toda razón, saber, en cambio, es equivalente a creer sobre la base de ciertas razones objetivas, pero no solamente en función de algún otro ocasional que me interrogue, sino también para mí mismo. No se trata del derecho del otro a desconfiar sobre mis asertos, sino que yo no puedo aceptar una creencia como saber sin esas razones objetivas. De ahí que también para mí saber involucra poner a prueba. La pregunta de Wittgenstein: "ise puede decir: 'donde no hay duda tampoco hay saber'?"30 parecería recibir una res-

29 Austin (1946) sostiene una analogía entre la jụtificación del uso de "yo 26 " y la del uso de "yo prometo". Por su parte, Hintikka, op. cit., p. 61, pone algunos reparos a esta analogía, al menos si se la pretende completa. Véase también Lehrer, op.cit., pp. $52-55$.

so Wittgenstein, op. cil., parágrafo 121. 
puesta afirmativa. Volveremos sobre ello. Ahora consideremos el caso de la certeza a la luz de estas nuevas observaciones.

Es oportuno reparar en un aspecto del planteamiento de Moore que aún no ha merecido nuestra atención. Como se recordará, la contingencia era una de las características comunes a todas las proposiciones afectadas por su declaración de certeza. Moore considera otras características, pero hay una que, sin ser mencionada aparte, atraviesa todo el trabajo: todas ellas son obvias. "Estoy en una habitación", "estoy de pie", "en mi mano tengo algunas hojas escritas", y otras por el estilo, ¿por qué estos ejemplos? Como dice Wittgenstein, "las verdades que Moore afirma saber son tales que, dicho sea de paso, si él las sabe, todos las sabemos". 31 Esto es lo que otorga a la declaración de certeza una fuerte dosis de razonabilidad, en la misma medida que ridiculiza a quien quiera dudar de la verdad evidente de lo afirmado. Sin embargo, la situación deja de ser ésta tan pronto como reemplazamos estas proposiciones por otras igualmente contingentes pero de ningún modo obvias, o si imaginamos que quien así habla lo hace bajo los efectos de un poderoso alucinógeno que lo induce a creerse de pie mientras está acostado, o que está en una habitación mientras toma sol en alguna playa. De esta forma, junto a la obviedad de esas afirmaciones desaparece aquello que en la certeza parecía un saber $y$, entonces, la creencia no fundamentada o aun falsa se manifiesta con toda crudeza. El tipo de proposiciones escogidas por Moore señala inmejorablemente la peculiaridad de la declaración de certeza: mientras que para el sujeto que la profiere no es necesario tener razones de ninguna clase - pues, ¿qué razón puede dar de, por ejemplo, "estoy cierto de que ahora estoy de pie"?, ¿acaso algo así: "porque lo siento"?-, los otros no tienen razones para dudar de su veracidad, pero, obviamente, no tener razones para dudar no implica sin más tener razones para afirmar. Es justamente el sentido de la expresión

31 Idem, parágrafo 100. Véase también el 462. 
"no sé si $p$ ", esto es, "no saber que $p$ y no saber que no $p$ ". La posición de Moore induce a caer en esta confusión, de tal modo que obtiene para su declaración de certeza los beneficios de la creencia y del saber, pero no sus desventajas. En efecto, la certeza expresada por Moore parece eximirnos del compromiso con razones objetivas como en el caso de la mera creencia; $y$, por otro lado, se asegura el consenso que s6lo es posible obtener a través de razones compartidas. Es el momento de retomar observaciones anteriores que nos permitirán captar con mayor precisión el contraste entre certeza y saber

Arriba afirmamos que "sólo cuando puedo dudar de mi certeza, ésta y mi saber no son lo mismo", y agregamos después que esto se volvía posible si existía el punto de vista de algún otro. Pero, ¿cuál es la situación ante proposiciones del tipo de las afirmadas por Moore? Respecto de ellas puede suponerse razonablemente que hay un acuerdo de todos los miembros pertinentes de la comunidad epistémica. ${ }^{32}$ ¿Qué se hace en este caso del punto de vista del otro?

Ante todo debemos preguntar: ¿para quién existe este todo imaginario? Sólo desde un punto de vista externo podemos juzgar algo como una totalidad. Soy yo, entonces, al imaginar o suponer el todo quien se ubica en el punto de vista del otro, y si, por el contrario, me incluyo como un miembro más de la comunidad epistémica, al abandonar el punto de vista externo ya no tengo ante mí una totalidad. Por lo demás, para este imaginario sujeto colectivo, vale la temporalidad tanto como para el sujeto individual. Luego, si la comunidad a la que nos referimos no tiene paradigma independiente con el cual medirse, más que una comunidad epistémica es una comunidad doxástica, en el sentido de que sus miembros comparten ciertas

32 Villoro, op. cit., propone que "Ilamemos 'sujeto epistémico pertinente' de la creencia de $S$ en $p$, a todo sujeto al que le sean accesibles las mismas razones que le son accesibles a $S$ y no otras, y 'comunidad epistémica pertinente', al conjunto de sujetos epistémicos pertinentes para una creencia" (p. 147). Es obvio que ahora todo el peso recae sobre las razones. El problema será identificarlas sin recurrir a los sujetos en cuestión. 
creencias básicas para las cuales no hay punto de vista desde el que puedan recibir un fundamento. Este es el significado de la observación de Wittgenstein: "en el fundamento de la creencia bien fundamentada se encuentra la creencia sin fundamentos". Por otra parte, estas creencias no tienen por objeto un conjunto definido de enunciados o proposiciones, sino un tipo de enunciados. Esto nos lleva a proponer un enunciado general como éste: "para cualquier comunidad epistémica, habrá siempre alguna clase de enunciados básicos sobre los cuales los miembros de la comunidad no pueden sostener ningún saber en sentido estricto, pues no hay ninguna instancia desde la cual podrían fundamentar sus creencias en la verdad de esos enunciados". Hay una hermosa imagen de Wittgenstein que parece dirigida a señalar lo mismo: "podríamos imaginar que algunas proposiciones, que tienen la forma de proposiciones empíricas, se solidifican y funcionan como un canal para las proposiciones empíricas que no están solidificadas y fluyen; y también que esta relación cambia con el tiempo, de modo que las proposiciones que fluyen se solidifican y las sólidas se fluidifican". 33 Y luego: "sí, el margen de aquel río es, en parte, de roca que no está sometida a ninguna alteración o que está sólo sometida a cambios imperceptibles, y, en parte, de arena que la corriente del agua arrastra y deposita en puntos diversos". ${ }^{34}$ Inmersos en el fluir de la corriente, no podemos pretender construir el lecho que permite el curso de ese fluir.

Metaforas aparte, estas reflexiones muestran que la declaración de certeza que un sujeto individual pudiera hacer en relación con esas proposiciones básicas, es más bien una parodia de saber que un saber. Sin embargo, y aun contra sí mismo, la posición asumida por Moore es extraordinariamente significativa y esclarecedora, pues al anunciar a título personal lo que toda la comunidad comparte, evidencia la falta de funda- 
mentación última de esas creencias comunes. Ahora bien, si el caso imaginario considerado no constituye un contraejemplo de la afirmación de que el saber supone la posibilidad del punto de vista del otro o, como dice Villoro, si "en la actualidad instantánea de mi conciencia propiamente nada sé", sino que "sólo tengo certezas", pues "para saber algo necesito salir de mí y compartir otro punto de vista", porque "el saber requiere la comunidad de un sujeto con otros", 35 entonces podemos expresar esta exigencia con el siguiente enunciado:

$$
\left(S_{Y} p\right) \rightarrow C_{Y}(\exists x)\left(P\left(-S_{x} p \cdot-S_{x}-p\right) \cdot(x \neq Y)\right),
$$

donde $x$ es cualquier otro sujeto lógico, sea otra persona o yo mismo en posición de otro respecto de mí, o bien imaginariamente, o bien como ocurre efectivamente respecto del pasado y del futuro. (Además, los sujetos lógicos son posiciones formales, no seres humanos.) Este enunciado intenta expresar una condición necesaria para mi saber: la existencia para mi de algún otro al que le sea posible (P) considerar a ' $p$ ' no-decidida, es decir, no saber si $p$. Literalmente, el enunciado puede leerse así: "si yo sé que $p$, entonces creo que existe un $x$ para quien es posible no saber que $p$ y no saber que no $p$, y $x$ no es idéntico a mî". Algunos aspectos de este enunciado merecen ser comentados.

En primer lugar, hemos usado ' $Y$ ' que, como se recordará, según nuestra convención simboliza el pronombre personal 'yo'. Lo hicimos así con el objeto de mostrar más fuertemente el contraste entre certeza y saber, ya que la declaración de certeza está hecha en primera persona. Sin embargo, no hay inconveniente en generalizar (6) así:

$$
\text { (7) }(z)\left(\left(S_{z} p\right) \rightarrow C_{z}(\exists x)\left(P\left(-S_{x} p \cdot-S_{x}-p\right) \cdot(x \neq z)\right)\right)
$$

La generalización universal evita aquí pensar que la base de esta condición es introspectiva, pues, desde luego, no se trata

Villoro, op. cit., p. 141. 
de que yo me percato por un peculiar acto de conciencia de que cada vez que sé algo, también creo que algún otro puede poner en duda mi saber. Por lo demás, 'saber' y 'ser consciente de' no son nociones equivalentes, aunque se relacionan de modo diverso.

En segundo lugar, es importante advertir la razón de incluir el operador ' $P$ ' allí donde se lo incluye. Si no lo hiciéramos así, se seguiría que en la situación hipotética de que todos los miembros de una comunidad epistémica coincidieran en la aseveración de ' $p$ ', de ninguno de ellos podría afirmarse que sabe que $p$, debido a que para nadie - supuesta esta situación como permanente- ' $p$ ' estaría sin decidir.

Finalmente, el aspecto más importante de esta proposición es que el consecuente entero esté precedido por el operador ' $C$ '. En efecto, saber que $p$ no implica la necesidad de una prueba independiente de la existencia del prójimo ni nada que se le parezca. Tampoco involucra un saber acerca de esa existencia de un punto de vista diferente del propio. Lo que la condición afirma es, simplemente, que sólo si para mi hay tal punto de vista soy capaz de saber alguna cosa con total legitimidad. Basta que lo crea para que esa supuesta alteridad funcione.

En forma general, (7) enuncia entonces una de las condiciones necesarias para saber. Pero además, esta condición tiene la particularidad de ser también necesaria para distinguir creencia de saber. A fin de comprender mejor la manera en que ha sido desarrollada esta distinción, será de gran ayuda ilustrarla históricamente con una referencia a Descartes. Cuando, después de haber logrado someter a duda todas las informaciones de orden sensible a través de la hipótesis del sueño y de consideraciones acerca de la naturaleza contradictoria de algunas de esas afirmaciones, Descartes repara en que hay, con todo, algunas verdades que insisten, y para poder extender a ellas su desconfianza sistemática debe recurrir al punto de vista de un otro que conozca la falsedad de tales proposiciones que él cree verdaderas, y que por lo tanto conozca cuáles son 
realmente las verdaderas. El lugar de este otro es el de Dios o el del hipotético "genio maligno". No importa ahora el hecho de que estas proposiciones sean o no de un tipo distinto al que nos referíamos con ' $p$ '. Lo importante es advertir que, para abandonar el lugar de la certeza, esto es, para poner en duda el enunciado "sé con certeza que $p$ ", Descartes necesita suponer la existencia de un otro que, en este caso, no solamente no sabe si $p$, sino que, lo que es más fuerte, sabe que no $p$, es decir, que ' $p$ ' es falsa. Es el momento escéptico en el que Descartes ve seriamente amenazada la posibilidad de encontrar la verdad buscada, momento que, como es sabido, sólo será superado a partir del cogito. Ahora bien, el cogito no es todavía un saber, sino una certeza. Esto puede apreciarse, por ejemplo, en el hecho de que Descartes evita entender el contenido de lo que le entrega el cogito en términos de "animal racional", porque este camino volvería a hacer posible la duda. Cuando más tarde quiera alcanzar un saber propiamente dicho, se verá llevado a probar la existencia de Dios, es decir, del punto de vista del otro. En otras palabras, en Descartes la existencia de un punto de vista distinto del propio es una condición necesaria del saber y de su diferencia con la certeza. ${ }^{36}$

Al neutralizar el estado de certidumbre involucrado en la declaración de Moore, la condición formulada por (6) o (7), y que llamaremos "exigencia del punto de vista del otro", produce la distinción entre creer y saber. $\mathrm{Si}$ analizamos la declaración de certeza de Moore en términos de creencia, suponemos ya la satisfacción de esa condición, pues, de lo contrario, el sujeto sigue identificando creer y saber, justamente bajo la forma de la certeza. Desde el punto de vista de quien afirma (1), cuando se acepta un punto de vista distinto del propio como condición de posibilidad del saber, la certeza, como grado máximo de convicción, deja su lugar a la creencia propiamente dicha. Es el sentido de frases como "no creo que $p$, lo se", donde 'creer' y

${ }^{36}$ Descartes, Meditaciones metafisicas I y II. Véase el comentario de Villoro, op. cit., Pp. 142-144. 
'saber' están claramente diferenciados. Aquí, creer que $p$ implica no estar seguro de la verdad de ' $p$ '. Contra lo que algunos autores han sugerido, ${ }^{37}$ éste es un significado de 'creer' que una meditación filosófica sobre estas cuestiones debe recoger. Lo que resulta de las reflexiones hechas arriba es que, para que cualquier sujeto pueda creer en sentido estricto, es decir, perder su certeza y distinguir creer de saber, debe poder dudar de la verdad de su creencia, debe admitir la posibilidad del error. Se presenta así otra manera de explicar la anomalía que introduce la declaración de certeza, pues ella pretende una relación con la verdad que excluya la posibilidad de error, respecto de proposiciones que, por su naturaleza lógica, pueden ser verdaderas o falsas. Por esta razón, (4) o (5) son teoremas de una lógica de la certeza, no de la creencia. Dijimos arriba que 'creer' se distingue de 'saber' porque mientras el primero no nos compromete con la necesidad de justificar nuestro juicio, el segundo sí lo hace. Ahora vemos que esto se deriva del hecho de que la creencia propone una relación con la verdad muy diferente de la que propone el saber. Aunque esta cuestión es conceptual y no de hecho, prestar atención al uso ordinario puede sernos de gran ayuda. Normalmente, cuando estoy seguro de la verdad de ' $p$ ', no digo 'sé que $p$ ' a menos que alguien ponga en duda mi aseveración de ' $p$ '; de lo contrario, simplemente me limito a afirmar ' $p$ '. En este sentido, la enunciación explícita de un saber parece redundante, mientras que no es redundante decir 'creo que $p$ ' en lugar de la mera aseveración de ' $p$ '.

Tenemos entonces la siguiente cadena de afirmaciones: para poder adquirir un saber debo renunciar a la inmediatez de una garantía de verdad estrictamente subjetiva como el estado de certeza, y esto se hace posible aceptando la existencia de un punto de vista diferente del propio, consistente en poder man-

37 Idem, p. 15 y Galván, op. cit., en el capítulo de su lógica ya citado (ver nota 23). Para una discusión de las relaciones entre 'creer' y 'saber' en este sentido, véase Lehrer, op. cit., capítulo III. 
tener como no-decidida la verdad de la proposición que es objeto de mi supuesto saber. Al neutralizarse de este modo la certeza, se posibilita la distinción entre creer y saber, en el sentido de que, mientras estar cierto excluye la posibilidad del error, afirmar solamente que creo en la verdad de determinado enunciado involucra per se admitir la posibilidad de equivocarme. A partir de esta secuencia, puede apreciarse la importancia que la posibilidad de dudar tiene para la constitución del saber. En efecto, la condición que llamamos "exigencia del punto de vista del otro" no muestra, en última instancia, otra cosa. Incluso podríamos decir que el otro del sujeto de la certeza es el escéptico. Es posible incluso sospechar que cierta interpretación de la posición escéptica pone en evidencia que el escéptico no es necesariamente una amenaza para las pretensiones de conocimiento fundado y que, por el contrario, al desestabilizar al sujeto de la certeza, al expulsarlo de su seguridad solipsista, es quien verdaderamente abre la posibilidad del saber. ${ }^{38}$

\section{REFERENCIAS BIBLIOGRÁFICAS}

Ambrose, Alice: "Moore's 'Proof of an External World'", en P. A. Schilpp (ed.), The Philosophy of G. E. Moore, Nueva York, 1952.

Galvan, Sergio: Introduzione alle Logiche Filosofiche I: Estensioni della Logica proposizionale classica, Vol. 1, Milán, 1985.

Hintikka, Jaakko: Knowledge and Belief, Cornell University Press, Nueva York, 1962.

Lehrer, Keith: Knowledge, Clarendon Press, Oxford, 1974.

Lenzen: Recent Work in Epistemic Logic, Acta Philosophica Fennica, Amsterdam, 1978.

Malcolm, Norman: "Moore and Ordinary Language", en el volúmen editado por Schilpp antes citado, y "Defending Common Sense", en The Philosophical Review, 1949.

Moore, George E.: Philosophical Papers, George Allen \& Unwin, Londres, 1959.

38 Para una revaloración de la perspectiva escéptica diferente de la aquí desarrollada, puede consultarse Unger, op. cit.. 
Stroud, Barry: The Significance of Philosophical Skepticism, Clarendon Preas, Oxford, 1984.

Unger, Peter: "A Defense of Skepticism", en Philosophical Review, 80, 1971 , pp. $198-219$.

Villoro, Luis: Creer, saber, conocer, Siglo XXI, México, 1982.

Von Wright: Ensayo de logica modal, Rueda, Buenos Aires, 1970.

Recibido: 22 mayo 1989. 


\section{SUMMARY}

Traditionally, the skeptic has been considered as a threat to our claims to true and justified knowledge. Also, certainty appears to be as the highest possible degree of knowledge. Knowledge and certainty are thus opposed to skepticism.

This paper wants to show that 'certainty' and knowledge are, probably, incompatible notions, and that the possibility of a doubt about the assumed certainty is a necessary condition to distinguish between belief and knowledge, and to construe any kind of knowledge.

Its starting point is Moore's notion of certainty. Moore demands that the expression of certainty (and, consequently, its formalization) should assume certain basic intuitions. One of them is the non-transmissibility of certainty; another intuition, fundamental to its Proof of the Existence of the World, is that certainty should be implied by knowledge.

The claim of certainty:

(1) "I know with absolute certainty that $p$ "

can be considered as a 'residual meaning' of the following formal expression in the epistemic logic of Hintikka:

(2) " $S_{Y} S_{Y} p$ ",

where ' $S$ ' is the epistemic operator which stands for knowledge, ' $Y$ ' is the personal pronoun ' $l$ ', and ' $p$ ' refers to the proposition claimed to be known. In other words, we would claim to know that we know a given proposition.

This proposal has some disadvantages: in Hintikka's view, it is virtually equivalent to ' $S_{Y} p$ ', that is, to 'I know that $p$ ', and, even if Hintikka went astray, because knowledge is transmissible, certainty would be transmissible too, and this contradicts Moore's intuition concerning the nontransmissibility of certainty.

Another interpretation of (1) is

(3) " $N S_{Y} p$ " ("I necessarily know that $p$ ")

where ' $N$ ' is the necessity operator. But this interpretation fails because it also contradicts the non-transmissibility, and intuitively it is very hard to believe that " $S_{Y} p \rightarrow N S_{Y} p$ ", that is to say, it is very hard to believe that it matches the intuition that knowledge implies certainty.

An alternative would consist on relating certainty and belief. If ' $C$ ' is the belief operator in epistemic logic, the following theorem by Galván comes close to Moore's demands:

(4) " $C_{Y}\left(C_{Y} p \rightarrow p\right)$ ",

that is, "I believe that I believe only truths". It can also be put as:

(5) " $C_{Y} p \rightarrow C S_{Y} p$ " ("If I believe that $p$, therefore I believe that I know that $p$ "). 
Unfortunately, neither (4) nor (5) are warrants of truth, but warrants of the impossibility of doubt. This is opposed to Moore's claim that we know that the premises of the Proof of the External World are undebatably true.

Luis Villoro has pointed out the necessity of taking into consideration the epistemic communities when we speak of knowledge. This requirement is stated thus:

(6) $\left(S_{Y} p\right) \rightarrow C_{Y}(\exists x)\left(P\left(-S_{x} p \cdot-S_{x}-p\right) \cdot(x \neq Y)\right)$,

which can be read as: "If I know that $p$, therefore I believe that there is an $x$ such that it is possible for him not to know that $p$ and not to know that $n o-p$, and $x$ is not identical with me". This can be generalized thus:

(7) $(z)\left(\left(S_{z} p\right) \rightarrow C_{z}(\exists x)\left(P\left(-S_{x} p \cdot-S_{x}-p\right) \cdot(x \neq z)\right)\right)$

Moreover, from this formalization it follows that a subject should be able to doubt about the truth of his belief: he must admit the possibility of error, and this conflicts with certainty.

In onder to be able to get knowledge I must abandon a strictly subjective warranty of truth, such as certainty, and this is possible when I accept the existence of a point of view which differs from mine, a point of view which lets undecided the truth or falsity of a proposition. In this way it is possible to distinguish between believing and knowing, because knowledge still demands the possibility of error.

So, there is a certain interpretation of skepticism that can see it not as a threat to the claims to justified knowledge, but as the position that truly offers the possibility of knowledge, because it fights the solipsistic assurance of the subject of certainty.

[Francisco Hernández] 